29. A. Davaille, F. Girard, M. Le Bars, Earth Planet. Sci. Lett. 203, 621 (2002).

30. P. Goldreich, A. Toomre, J. Geophys. Res. 74, 2555 (1969).

31. J. Besse, V. Courtillot, J. Geophys. Res. 107, 10.1029/ 2000JB000050 (2002).

32. J. A. Tarduno, A. V. Smirnov, Earth Planet. Sci. Lett. 184, 549 (2001).

33. J. A. Tarduno, A. V. Smirnov, Earth Planet. Sci. Lett. 198, 5533 (2002).

34. R. A. Livermore, F. J. Vine, A. G. Smith, Geophys. J. R. Astron. Soc. 79, 939 (1984).

35. R. Van der Voo, T. H. Torsvik, Earth Planet. Sci. Lett. 187, 71 (2001).
36. D. C. Engebretson, A. V. Cox, R. G. Gordon, Geol. Soc Am. Spec. Pap. 206 (1985), pp. 1-59.

37. H.-P. Bunge, S. P. Grand, Nature 405, 337 (2000).

38. M. A. Richards, C. Lithgow-Bertelloni, Earth Planet. Sci. Lett. 137, 19 (1996).

39. J. A. Tarduno, J. Gee, Nature 378, 477 (1995).

40. R. D. Mueller, W. R. Roest, J.-Y. Royer, L. M. Gahagan, J. G. Sclater, J. Geophys. Res. 102, 3211 (1997).

41. This research used samples provided by the ODP, which is sponsored by NSF and participating countries under management of the Joint Oceanographic Institutions. We thank the scientific and operational parties of Leg 197 for their contributions at sea; the staff of ODP-Texas A\&M University for their techni- cal support; and P. Doubrovine, G. Olton, D. Sinnett, and A. O'Kane for assistance with magnetic measurements. Supported by NSF.

Supporting Online Material

www.sciencemag.org/cgi/content/full/1086442/DC1

Materials and Methods

Figs. S1 and S2

Tables $\mathrm{S} 1$ to $\mathrm{S} 5$

5 May 2003; accepted 8 July 2003

Published online 24 July 2003;

$10.1126 /$ science. 1086442

Include this information when citing this paper.

\section{Hairpin RNAs and Retrotransposon LTRs Effect RNAi and Chromatin-Based Gene Silencing}

\author{
Vera Schramke and Robin Allshire*
}

\begin{abstract}
The expression of short hairpin RNAs in several organisms silences gene expression by targeted mRNA degradation. This RNA interference (RNAi) pathway can also affect the genome, as DNA methylation arises at loci homologous to the target RNA in plants. We demonstrate in fission yeast that expression of a synthetic hairpin RNA is sufficient to silence the homologous locus in trans and causes the assembly of a patch of silent Swi6 chromatin with cohesin. This requires components of the RNAi machinery and Clr4 histone methyltransferase for small interfering RNA generation. A similar process represses several meiotic genes through nearby retrotransposon long terminal repeats (LTRs). These analyses directly implicate interspersed LTRs in regulating gene expression during cellular differentiation.
\end{abstract}

Eukaryotic genomes invariably contain repetitive DNA sequences of uncertain functional significance: large expanses of heterochromatin are associated with centromere regions and flank the kinetochores at centromeres. This centromeric heterochromatin is frequently made up of tandem arrays of simple satellites interspersed with transposable elements (1-5). Moreover, recent studies support a direct role for such tandem repeats in centromere function, because arrays of human centromeric $\alpha$-satellite allow de novo kinetochore assembly (1). In addition, the heterochromatin that coats centromeric repeats in fission yeast (Schizosaccharomyces pombe) plays an important role in sister-centromere cohesion $(6,7)$.

Other repetitive elements (e.g., retrotransposons) are found scattered throughout eukaryotic genomes (8). It is becoming apparent that interspersed repeats can influence the expression of nearby genes $(9,10)$. McClintock first recognized this potential role for transposons in maize and referred to them as controlling elements (11). Consistent with this, mutations that reduce DNA methylation in plants result in activation and mobilization of transposons and lead to epimuta-

Wellcome Trust Centre for Cell Biology, Institute of Cell and Molecular Biology, King's Buildings, University of Edinburgh, Edinburgh EH9 3JR, Scotland, UK.

*To whom correspondence should be addressed. Email: robin.allshire@ed.ac.uk tions $(12,13)$. Although transposable elements can alter the expression of neighboring genes in plant and animal cells $(8-10)$, it remains unclear whether these elements play a direct role in endogenous gene regulation. Nevertheless, early models proposed that families of interspersed repetitive sequences might regulate networks of genes and lead to cellular differentiation in response to a signal (14).

The heterochromatic nature of centromeres and the abundance of transposable elements at pericentromeric regions in some organisms suggests links between transposon silencing and centromeric heterochromatin formation. In mammalian cells, both centromeric and retrotransposon repeats have a similar repertoire of both DNA and chromatin modifications that are usually found associated with silent chromatin and also attract cohesin (15-18)

Fission yeast centromeres are composed of outer repeats that flank the central kinetochore domain. Marker genes placed within these repeats are silenced by a process requiring Clr4, which methylates histone $\mathrm{H} 3$ on lysine 9 (MeK9-H3), which allows binding of Swi6 (the ortholog of metazoan HP1) and Chp1 to these outer repeats (19-24). Overlapping noncoding transcripts are produced from these repeats, and analyses suggest that their processing by the RNA interference (RNAi) machinery results in the generation of small interfering RNAs (siRNAs), which are continually required for the formation of silent chromatin over these centromere repeats $(25,26)$ and for centromere function (27-29). Proteins related to CENP-B and the mariner class of transposases also bind the outer repeat sequences at fission yeast centromeres and contribute to the formation of heterochromatin (30). In addition to centromeric repeats, the standard fission yeast genome contains 13 intact Tf2 retrotransposable elements plus $249 \mathrm{Tf1}$ and Tf2 long terminal repeats (LTRs), or related LTRs, and LTR fragments dispersed throughout the genome (31).

In many organisms, the process of RNAi has been exploited to nullify gene expression (32). Silencing can occur in two ways: posttranscriptional gene silencing (PTGS) involves degradation of the targeted RNA; studies in plants indicate that expression of double-stranded RNAs (dsRNAs) can result in DNA methylation and transcriptional gene silencing (TGS) at the target locus (33-35). Moreover, the RNAi pathway is required for the generation of siRNAs homologous to, and DNA methylation of, retrotransposons in plants $(36,37)$. Here we test whether, in fission yeast, the RNAi machinery is preserved to specifically process transcripts generated from centromeric repeat sequences or if it can act to silence noncentromeric transcripts in trans and trigger heterochromatin formation. We also demonstrate that endogenous, developmentally regulated, lineage-restricted genes are subject to control by this same process and that adjacent retrotransposon LTRs effect this regulation.

Short hairpin RNA (shRNA) can silence a normally expressed ura4 ${ }^{+}$gene. To determine whether the RNAi machinery of fission yeast can act to process noncentromeric repeat transcripts, we cloned an internal region [280 base pairs (bp) of $\mathrm{ura}^{+}$, Stu I-Eco RV (SE)] from the ura4 open reading frame (ORF) in an inverted orientation around a 355-bp spacer under the control of the $n m t 41$ promoter (shuraSE; Fig. 1A). To assess whether expression of shuraSE could induce silencing of the $\mathrm{ura}^{+}$ gene, we transformed the construct and empty vector into two strains; one in which the endogenous $\mathrm{ura}^{+}$locus remained intact and the other in which the 280-bp Stu I-Eco RV region was deleted from the ura4 gene (ura4-DS/E, which lacks homology to shuraSE) and a wild-type copy of $\mathrm{ura}^{+}$had been inserted at another location (Rint:ura4 ${ }^{+}$). Previous analyses demon- 


\section{RESEARCHARTICLES}

strate that Rint:ura4 ${ }^{+}$is expressed at the same levels as the truncated, nonfunctional transcript arising from $u r a 4-D S / E$ in the same cells and is never silenced (38). Cells expressing $\mathrm{ura}^{+}$ grow on medium lacking uracil (-URA) but are unable to grow on counter-selective plates containing 5-fluoro-orotic acid (FOA). Conversely, silencing of $\mathrm{ura}^{+}$inhibits growth on medium lacking uracil but allows growth on FOA. Expression of shuraSE blocked growth of both strains Rint:ura4 ${ }^{+}$and the normal $\mathrm{ura}^{+}{ }^{+}$locus; also $\mathrm{ura}^{+}$inserted at eight other distinct locations (39) on medium lacking uracil and allowed colony formation on FOA plates (Fig. 1B). The level of Rint:ura $4^{+}$transcription was significantly reduced relative to the ura4-DS/E internal control (Fig. 1C). This demonstrates that only sequences homologous to the shRNA $\left(\mathrm{ura}^{+}{ }^{+}\right.$not ura4-DS/E) are targeted for silencing.

shRNA silencing of ura4 $^{+}$requires RNAi and heterochromatin components. Argonaute, Dicer, and RNA-directed RNA polymerase act to mediate RNAi in many organisms (32). The S. pombe homologs Ago1, Dcr1, and Rdp1 are required for both silencing and the assembly of silent chromatin over outer centromere repeats (26). Expression of shuraSE in strains with deletions of $\mathrm{agol}^{+}, \mathrm{dcrl}^{+}$, or $\mathrm{rdpl}^{+}(\operatorname{agol} \mathrm{A}$, $d c r 1 \Delta$, or $r d p 1 \Delta$ ) did not induce silencing of Rint:ura $4^{+}$(Fig. 1, B and C). Thus, silencing of Rint:ura $4^{+}$by shuraSE expression is dependent on RNAi components, consistent with silencing by PTGS.

To determine whether known factors required for chromatin-based transcriptional silencing affect shuraSE mediated silencing, we also introduced the constructs into cells containing Rint: $\mathrm{ura4}^{+}$, which lack Clr4 K9-H3 methyltransferase function or the HP1 ortholog, Swi6. Because these are chromatin-based silencing factors these lesions are not expected to affect PTGS. Surprisingly, phenotypic silencing of $\mathrm{urat}^{+}$was completely abolished in $\operatorname{clr} 4-s 5$ (no growth on FOA) but persisted in swi6s cells (Fig. 1, B and C). $\mathrm{ura}^{+}$transcript levels remained relatively low in swi6s cells as compared to clr4-s5 and $d c r 1 \Delta$ cells expressing shuraSE. The fact that clr4-s5 lacks silencing of $\mathrm{ura4}^{+}$indicates that RNAi is defective and suggests that, apart from methylating histone $\mathrm{H} 3, \mathrm{Clr} 4$ acts in the PTGS pathway. Consistent with this siRNAs homologous to shuraSE are not detected in clr4-s5, in addition to agols, $d c r 1 \Delta$, or $r d p 1 \Delta$ cells, but are generated in swi6s cells (Fig. 1D). Thus, the RNase III endonuclease Dcrl requires Ago1, Rdp1, and Clr4 to attack dsRNA and to generate siRNAs that target endogenous $\mathrm{ura}^{+}$transcripts for degradation.

shura4 expression recruits silent chromatin modifications and proteins to ura $4^{+}$. In wild-type fission yeast, the RNAi pathway generates siRNAs homologous to centromeric repeats that then induce the degradation of the corresponding transcripts. Modification of chromatin [dimethyl-K9-H3 (diMeK9-H3) and Swi6 binding] over the centromeric repeats appears to be coupled to this process $(25,26)$. The requirement of Clr4 for shuraSE-mediated silencing suggested that chromatin modifications might be transmitted to the target locus.

To test whether expression of shuraSE leads to chromatin modifications at the ura 4 locus, we used chromatin immunoprecipitation (ChIP) to assess the methylation status of histone $\mathrm{H} 3$ (diMeK9-H3 and diMeK4-H3) and Swi6 association at the Rint:ura4 ${ }^{+}$locus compared with the ura4-DS/E internal control in wild-type, agols, $d c r 1 \Delta, r d p 1 \Delta, c l r 4-s 5$, and swi6 $\Delta$ cells (Fig. 2A). In wild-type cells, expression of shuraSE resulted in accumulation of diMeK9-H3 and recruitment of Swi6 at the Rint:ura4 ${ }^{+}$locus but not at the ura4-DS/E locus, which lacks homology with shuraSE. Dimethyl-K9-H3 and Swi6 association was not detectable at Rint:ura4 ${ }^{+}$in agols, $d c r 1 \Delta, r d p 1 \Delta$, or clr4-s5 cells compared with wild-type cells; instead, the diMeK4-H3 mark, normally associated with active chromatin, remained comparable to that detected in wild-type cells not expressing shuraSE. In contrast, in swi6s cells, diMeK4-H3 persisted even though diMeK9-H3 is still induced by shuraSE at Rint: $\mathrm{ura}^{+}$(Fig. 2A). This pattern of modifications is identical to that seen at centromeric repeats in swi6s cells $(22,24,29)$. Further analyses dem- onstrate that diMeK9-H3 is also detectable at the $\mathrm{ura}^{+}$promoter and between the promoter and the target uraSE region of the silenced Rint: $\mathrm{ura}^{+}$gene (Fig. 2B). Thus, silent chromatin can spread outward from the targeted uraSE region (see last figure). In the absence of Swi6, diMeK9-H3 is no longer detected at the promoter. Thus, as at the silent mating type locus (40), Swi6 is required to spread silent chromatin outward from the nucleation site, which, in this case, is the 280-bp uraSE region homologous with the shRNA.

Cohesin is also known to bind Swi6containing chromatin $(6,7)$. ChIP was performed on a strain expressing hemagglutinin (HA) tagged $\operatorname{Rad} 21$ and shuraSE. Rad21 cohesin is also associated with shRNA-induced heterochromatin over the Rint:ura4 ${ }^{+}$gene (Fig. 2C). Thus, the expression of an shRNA is sufficient to induce the assembly of silent chromatin at a normally euchromatic locus, and this silent chromatin has all the hallmarks and features of bona fide fission yeast centromeric heterochromatin.

Meiotic genes are up-regulated in cells lacking RNAi or heterochromatin. Because shuraSE RNA expression results in repression of $\mathrm{ura}^{+}$by coupled posttranscriptional events and chromatin modifications, it seemed likely that endogenous genes

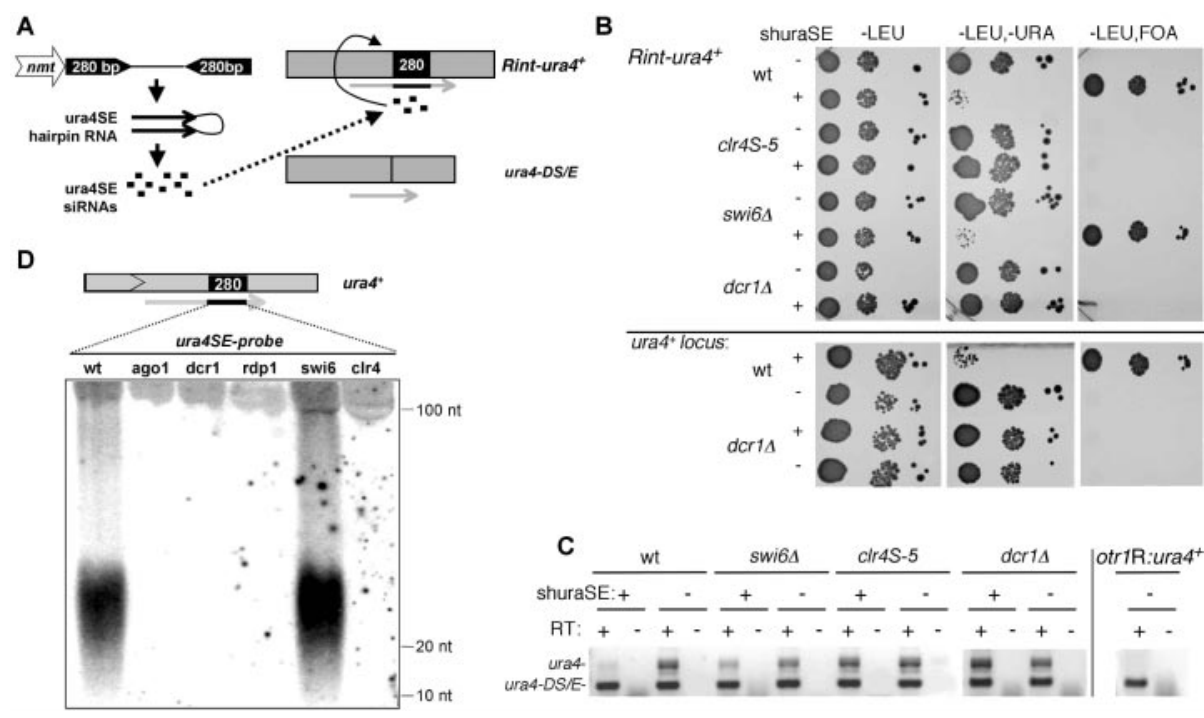

Fig. 1. An shRNA homologous to the normally expressed $u r a 4^{+}$gene induces silencing of $u r a 4^{+}$. (A) Expression of uraSE shRNA should generate siRNAs homologous to the 280-bp uraSE region of Rint:ura $4^{+}$gene but not the ura4-DS/E minigene. Rint:ura4 $4^{+}$but not ura4-DS/E mRNA may be targeted for degradation (dotted line) perhaps resulting in chromatin modifications (curved line). shuraSE is expressed from nmt41 on PREP41 (54). (B) Comparative growth assay of serially diluted strains transformed with pRep41-shuraSE $(+)$ or empty pREP41 $(-)$ were plated on selective media (-LEU), lacking uracil (-LEU, -URA) or containing 5-FOA (-LEU, FOA) (54). ( Top) Serial dilution of the wild-type (wt), clr4-s5, swi6s, and dcr1 1 , strains containing Rint:ura $4^{+}$and ura4-DS/E. (Bottom) Serial dilution of wild-type and dcr1 $1 \Delta$ strains with an intact ura $4^{+}$locus (39). (C) RT-PCR analysis of ura $4^{+}$transcripts was performed on oligo(dT)-primed complementary (CDNA) from RNA samples of the indicated strains in the presence $(+)$ or absence $(-)$ of reverse transcriptase (RT). Expression of Rint:ura $4^{+}$relative to the ura4-DS/E minigene compared with (+) or without (-) shuraSE. A strain with silenced ura $4^{+}$at cen 1 (otrIR:ura $4^{+}$) is included for comparison (19). ago1 $1 \Delta$ and $r d p 1 \Delta$ have the same effect as $d c r 1 \Delta$ and clr4-s5 (not shown). (D) Detection of shuraSE homologous siRNAs. Small RNAs were enriched from the indicated strains separated and transferred to filters. Labeled uraSE riboprobe was hybridized, and filters were washed and exposed to obtain this phosphorimage. Relative size was determined from markers in neighboring tracks. 
might also be regulated by RNAi-mediated chromatin modifications.

In rich growth media, mitotically dividing fission yeast cells repress a battery of genes that

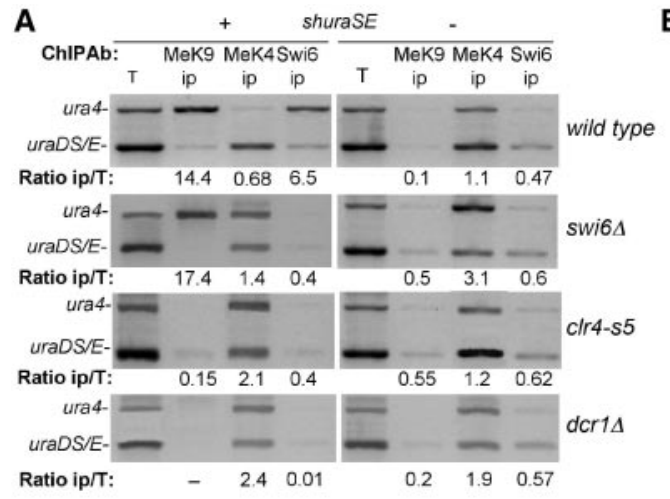

are induced after nitrogen starvation, which sets off sexual differentiation, resulting in conjugation, zygote formation, and meiosis $(41,42)$. One study identified 31 cDNAs that were con-

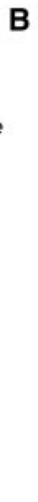

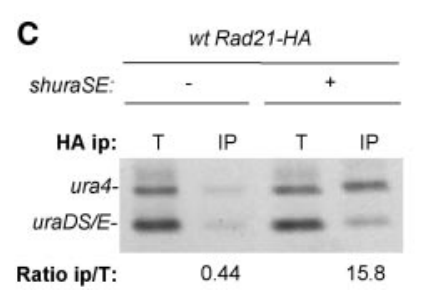

ura $4^{+}$is compared with the internal control ura4-DS/E. Similar data were obtained in three independent experiments. (B) ChIP samples prepared from wild-type and swi6 $\Delta$ cells expressing shuraSE were used to detect diMeK9-H3 over the ura4SE region (400-bp PCR product, p 1); the $5^{\prime}$ adjacent region (300-bp product, p2); and the $u r a 4^{+}$promoter (pro; 250-bp product, $\mathrm{p} 3)$. The $\mathrm{p} 1$ primers also amplify a smaller product from the ura4-DS/E allele as an internal control. Primers that amplify $200 \mathrm{bp}$ from act $1^{+}$were used as an internal control with p2 and p3 primers. (C) HA-specific antibodies were used to immunoprecipitate chromatin from wild-type cells bearing 3xHA epitope-tagged $\mathrm{rad}_{21^{+}}$at the endogenous locus, the euchromatic Rint:ura $4^{+}$insertion, and the ura4-DS/E allele. Cells expressing $(+)$ or not expressing $(-)$ shuraSE were examined. Enrichment of ura 4 relative to ura4-DS/E was reproducible. firmed to be induced by nitrogen starvation (42). We noticed that 14 of these induced genes reside in close proximity (within $10 \mathrm{~kb}$ ) of a retrotransposon LTR (see Fig. 3A). Microarray analyses demonstrated that 700 genes are induced at levels at least 5 times higher after nitrogen starvation, meiosis, and sporulation, and some of these reside close to LTRs (41). Because plant retroelements have been shown to be targets for RNAi and are associated with MeK9-H3 (36, 43), we suspected that LTRs might play a role in repression of nearby genes during vegetative growth. We therefore used reverse transcription-polymerase chain reaction (RT-PCR) to test whether meiotically induced genes remain repressed in rich medium $(\mathrm{R})$ in wild-type, agol $1 \Delta$, dcr $1 \Delta, r d p 1 \Delta, c l r 4-s 5$, and swi6 $\Delta$ cells. We examined expression of four meu genes (mеи6, теи19, теи28, and mеи 30) and seven genes (ste7, SPAC30D11.02c, SPAC26H5.11, SPAC56F8.14c, SPAC56F8.17c, $m f r l, f b p l)$ shown by microarray analyses to be considerably induced during sexual differentiation. Each gene and its position relative to LTRs is depicted in Fig. 3A.

Analyses by RT-PCR confirmed that these eleven genes are induced after nitrogen starvation $(-\mathrm{N})$ of a homothallic [self-mating $\left(h^{90}\right)$ ] wild-type strain (Fig. 3B). Further analyses show that the meи6, mеи19, mеи28, ste7, SPAC30D11.02c (D11.02), SPAC26H5.11 (H5.11), and SPAC56F8.14c (F8.14) genes, whose promoters reside within $7 \mathrm{~kb}$ of an LTR, are derepressed in agol $\Delta, \operatorname{dcr} 1 \Delta$, $r d p 1 \Delta$, clr4-s5, and swi6s backgrounds of a heterothallic [not self-mating $\left(h^{-}\right)$]

Fig. 3. Meiotic genes residing in close proximity to LTRs are derepressed in cells lacking RNAi, clr4 $4^{+}$, or swi6 ${ }^{+}$. (A) Schematic representation of different meiotic genes (white rectangle) and their position relative to LTRs (light gray rectangle). Black arrowheads indicate the direction of gene transcription. The induction level determined in pat 1 synchronous meiosis by microarray analysis is shown in brackets (41). Distance (in kilobases) of the LTRs from the $5^{\prime}$ end of each ORF examined is indicated. (B) RT-PCR analysis was performed on oligo(dT)primed CDNA from RNA

A Identified as meiotic expression upregulated: Close to Tf1 or TF2 related LTR:

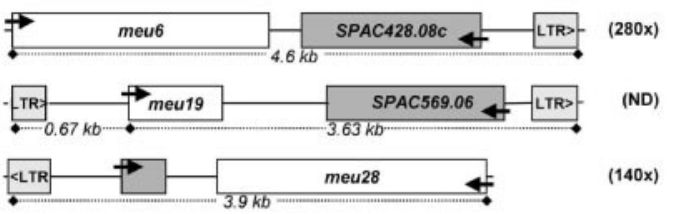

Induced during sexual differentiation (microarray):

Close to Tf1 or TF2 related LTR:
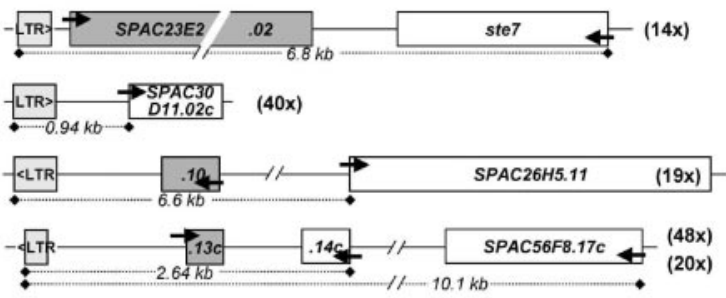

Far from Tf1 or TF2 LTR:

$-L T R>50 \mathrm{~kb}=-\mathrm{meu} 30$

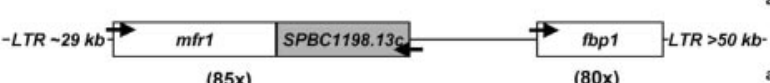

B

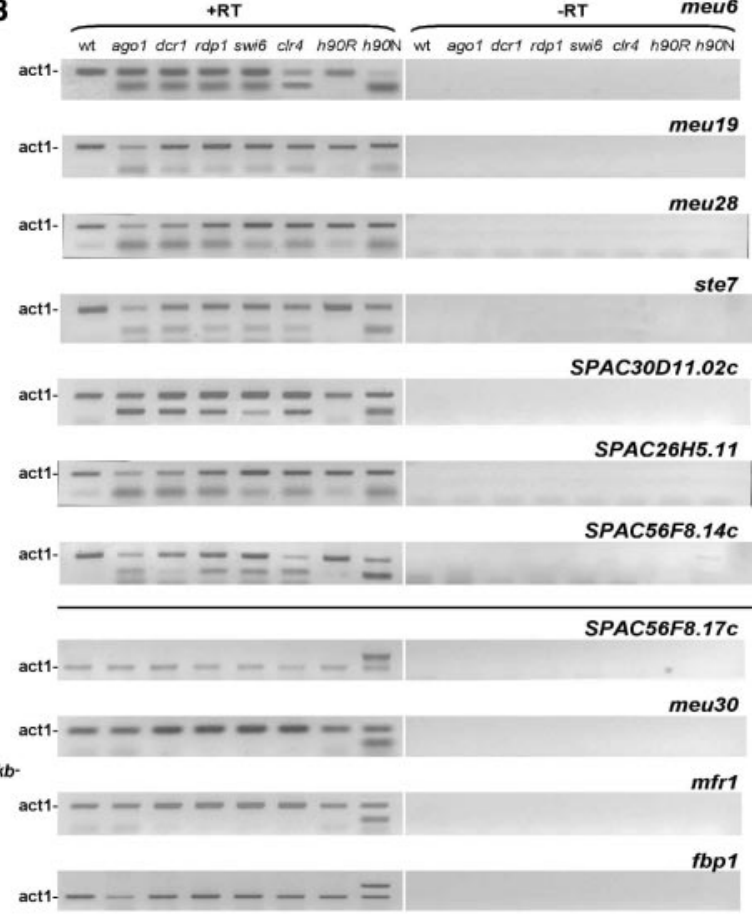
without ( $)$ revise transcriptase (RT). Expression of different transcipts was assessed by multiplex PCR, by comparing them to the constitutively expressed act $1^{+}$gene. All meiotic genes are induced in a homothallic $\left(h^{90}\right)$ wild-type strain after nitrogen starvation $\left(h^{90} \mathrm{~N}\right)$ but not when grown in rich medium $\left(h^{90} \mathrm{R}\right)$. SPAC56F8.17c and fbp 1 PCR products migrate above, whereas all others migrate below, the act $1^{+}$product. 


\section{RESEAR C H A T I C LES}

strain (Fig. 3B). In contrast, repression of meu30, $m f r l$, and $f b p l$, which are at least $29 \mathrm{~kb}$ away from an LTR, was unaffected in cells lacking Ago1, Dcr1, Rdp1, Clr4, or Swi6. In all cells and conditions, actin $\left(a c t 1^{+}\right)$transcripts were clearly detected. Repression of SPAC56F8.17c (F8.17), the promoter of which lies $7.5 \mathrm{~kb}$ distal to that of the responding $F 8.14$ gene and $10.1 \mathrm{~kb}$ from the same LTR, was unaffected by loss of Agol, Dcr1, Rdp1, Clr4, or Swi6 function.

Although not comprehensive analyses, these data show a compelling correlation between the proximity of an LTR and RNAi/ Swi6-dependent regulation of a gene. Surveying the $S$. pombe gene database (44) indicates that not all meiotically induced genes reside close to an LTR and that not all LTRs are found close to genes induced during sexual differentiation. However, our data suggest that, in some cases, retrotransposon LTRs may be required to maintain repression of nearby meiotically in- duced genes during vegetative growth. Other LTRs may act to enforce repression of genes normally induced under other conditions.

LTRs are coated with silent chromatin. The above analyses implicate Tf1- and Tf2related LTRs in the repression of nearby genes by RNAi-dependent chromatin-based silencing. To further assess this, we examined two LTRs to determine whether they are packaged in chromatin methylated on lysine 9 of histone $\mathrm{H} 3$ and bound by Swi6. ChIP assays indicated that, in wild-type cells, both LTRs were found to be coated by diMeK9-H3 chromatin and associated with Swi6 (Fig. 4). This modification and association were lost in the absence of Ago1, Dcr1, $\mathrm{Rdp1}$, or Clr4, whereas swi6s retained diMeK9H3. (We found similar results for LTR-meu6; only wild type and $d c r l \Delta$ are shown.) This suggests that the effects on D11.02 and meu6 gene expression are mediated by RNAi-dependent modifications at these LTRs.
LTRs are transcribed and required to mediate gene repression. Intact LTRs contain the promoter that in a complete retrotransposon drives transcription of the transposon ORFs (45). Solo LTRs may interfere with expression of nearby genes by driving expression of noncoding RNAs, which triggers RNAi-dependent silent chromatin assembly. To test this, we used primers to detect RNA derived from both strands across the meu $6^{+}$and D11.02 LTRs by RT-PCR (Fig. 5A). No transcript could be detected in wild-type cells; however, in agols, $d c r 1 \Delta, r d p 1 \Delta$, and clr4-s5 cells, transcripts from both the top and bottom strands accumulated, whereas the bottom transcript was predominantly detected in swi $6 \Delta$ cells. We surmise that, as with fission yeast centromere transcripts and shuraSE, these LTR transcripts are usually processed by the RNAi pathway and form siRNAs and that this process results in the modification of chromatin at the LTR which
A

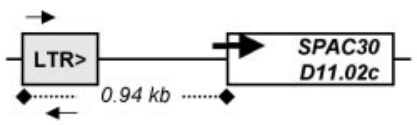
$\frac{\text { wild type }}{\text { Swi6 MeK9 }} \frac{\text { ago14 }}{\text { Swi6 MeK9 }} \frac{\text { dcr1s }}{\text { Swi6 MeK9 }}$
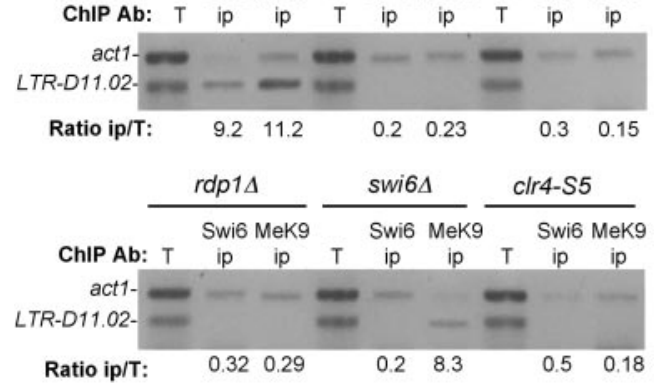

B
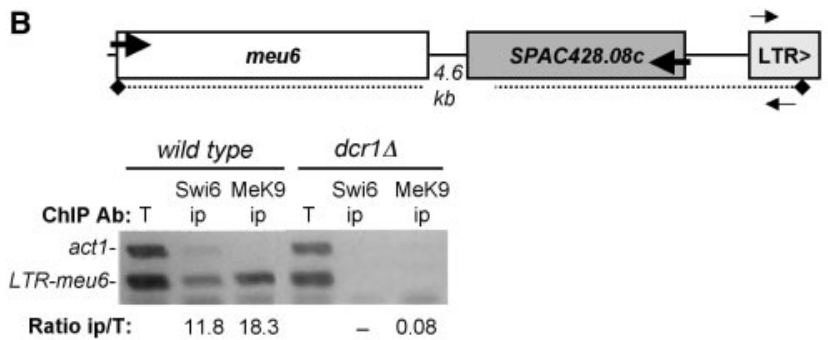

Fig. 4. LTR chromatin containing diMeK9$\mathrm{H} 3$ is associated with Swi6, and this requires RNAi, $\mathrm{clr}^{+}$, and swi6 ${ }^{+}$. ChIPs with antibodies recognizing dimethyl-K9-H3 and Swi6 were performed as described in Fig. 2A on wildtype or mutant cells.

We assessed enrichment of dimethyl-K9-H3 and Swi6 on the LTR region by multiplex PCR using primers (indicated by small black arrows) specific for the LTRs adjacent to D11.02 (A) and meu6 (B), respectively (lower bands). Primers that amplify 200 bp from act $1^{+}$were included as an internal control. LTR-meu6 primers gave similar results to those of LTR-D11.02 in ago1 $r d p 1 \Delta, c l r 4-s 5$ and swi6s (not shown).

Fig. 5. LTRs are transcribed, and they enforce repression of nearby meiotically induced genes. (A) Strand-specific transcript analyses at D11.02 and meu6 LTRs. Diagrams show the position of the LTRs relative to the meiotically up-regulated genes. Transcription direction of meiotic genes is indicated by large black arrows. The top (LTR for) or bottom strand (LTR rev) primers (small black arrows) were used to initiate CDNA synthesis; subsequently, the other primer was added to PCR amplify the prod-

A LTR for $\rightarrow$
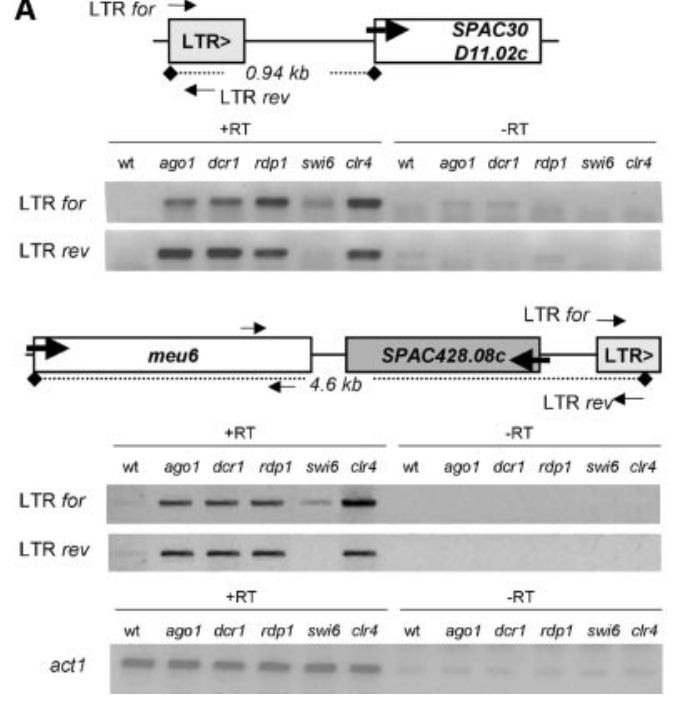
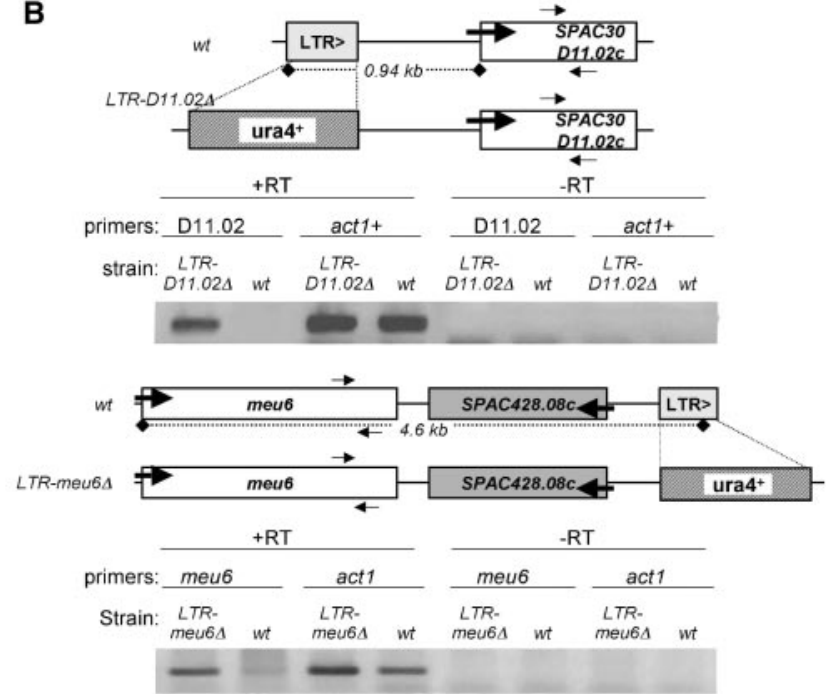

uct. RT-PCR analysis of LTR-specific transcripts from LTRs close to the D11.02 and meu6 genes was performed on LTR for or LTR rev primed cDNA from RNA samples of the indicated strains with $(+)$ or without $(-)$ reverse transcriptase (RT). Using PCR, 150 bp was amplified with LTR for and LTR rev primers specific for the D11.02 and meu6 LTRs. Amplification of $200 \mathrm{bp}$ of act1 from the same RNA samples was included as a control. (B) Replacement of the D11.02 and meu6 LTRs by the ura $4^{+}$. Diagrams show the generation of the LTR-D11.02 $\Delta$ and LTR-meu6s strains. The position of primers in D11.02 and meu6 are indicated (small black arrows). RT-PCR analysis of the D11.02 and meu6 transcripts was carried out in the indicated strains as described in Fig. 3B. Primers specific for act $1^{+}$were used as positive controls. 


\section{RESEARCHARTICLES}

can silence nearby genes. Loss of Ago1, Dcr1, Rdp1, Clr4, or Swi6 allows accumulation of the LTR transcripts and expression of the neighboring genes. Curiously, the bottom transcript preferentially accumulates in cells lacking Swi6, and this is reminiscent of the pattern of centromere transcript accumulation $(26,29)$.

To determine whether these LTRs are directly required for repression of nearby meiotically induced genes, we replaced the LTRs lying 0.94 $\mathrm{kb}$ upstream and $4.6 \mathrm{~kb}$ downstream of the D11.02 and meu6 gene promoters, respectively, with the $\mathrm{ura}^{+}$gene by homologous recombination by sequences immediately flanking the LTRs to generate LTR-D11.02A and LTRmeu6 $\Delta$. During vegetative growth conditions,

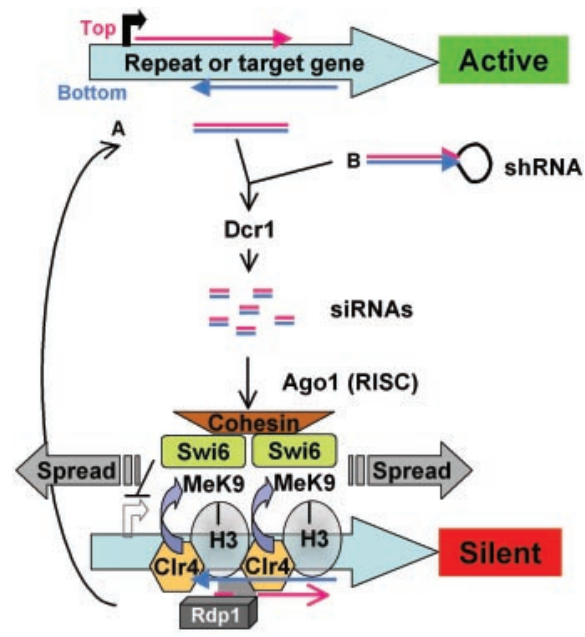

Fig. 6. Model for the nucleation and spreading of silent chromatin from centromeric repeats, LTRS, or an shRNA target. Top and bottom strand noncoding transcripts from retrotransposon LTR or centromeric repeat DNA overlap, hybridize, and form dsRNA (A). Expression of synthetic shRNAs also allows formation of dsRNA homologous to a target gene (B). dsRNAs are cleaved to siRNAs by Dicer (Dcr1); a putative complex containing Ago 1 (RISC) binds these siRNA and uses them to target homologous nascent transcripts at the target locus. Rdp1 (RNA-directed RNA polymerase) may use siRNAs to back-transcribe this transcript, and in the process, Clr4 methylates lysine 9 of histone $\mathrm{H} 3$ on the surrounding chromatin, which allows binding of Swi6, the formation of silent chromatin, and recruitment of cohesin $(26,55)$. Transcription from the top strand of centromere repeats or the shRNA target gene is repressed. Transcription and RNAi-mediated degradation of the bottom strand of centromeric repeats and LTR transcripts continues even after assembly of Swi6 chromatin (26). Rdp1 activity ensures that homologous dsRNA and siRNAs are continually generated, and thus, silent chromatin is maintained over these repeats. Direct recruitment of Clr 4 by assembled Swi6 chromatin may allow this to spread along the chromatin fiber and may mediate silencing of genes adjacent to LTRs. Alternatively, noncoding transcripts originating in the LTR that extend into adjacent genes may trigger the formation of siRNAs homologous to that gene, which results in its silencing. the D11.02 and meu6 RNAs were constitutively

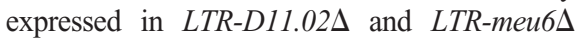
cells (Fig. 5B). This indicates that the LTR lying adjacent to the D11.02 gene and the LTR separated by another ORF $3^{\prime}$ to the meu 6 gene play a direct role in the repression of these genes during vegetative growth in rich medium. We conclude that LTRs can play a critical role in restricting the expression of a gene to a distinct differentiation pathway and that components of the RNAi pathway and silent chromatin are required to effect this repression.

Discussion. Our data indicate that expression of a synthetic shRNA can silence expression of a euchromatic gene in trans and this is coupled to chromatin modification and recruitment of heterochromatin proteins and cohesin to the target locus. Thus, expression of a shRNA can, alone, trigger the assembly of a patch of silent chromatin, which mimics that associated with the centromeric outer repeats.

The synthetic shuraSE hairpin RNA trigger requires Argonaute, Dicer, and RNA-directed RNA polymerase, the known components of the RNAi machinery, to mediate silencing. Thus, the RNAi machinery of fission yeast is not just designed to process unusual centromeric transcripts specifically, but, as in other organisms, it appears to degrade any extensive dsRNA. A surprising finding was that expression of shuraSE RNA also requires functional $\mathrm{Clr} 4$ to produce shuraSE siRNAs and to reduce $\mathrm{ura}^{+}$expression; however, phenotypic silencing of ura4 (FOA resistance), siRNA generation, and diMeK9-H3 over the $u r a S E$ region all persist in the absence of Swi6, even though ura4 RNA levels appear elevated. This suggests that Swi6 operates downstream of the PTGS (RNAi) pathway to mediate transcriptional silencing at the level of chromatin. Because gene silencing is completely ineffective in clr4-s5, this histone methyltransferase is required both for efficient PTGS and the downstream modification of histone $\mathrm{H} 3$ on lysine 9 to mediate TGS. It seems likely that Clr4 acts in concert with Dicer, Ago1, and Rdp1 and that, in its absence, complexes [for example, RISC; see (41)] that are required for RNA interference are disrupted (see Fig. 6).

In wild-type cells expressing shuraSE, diMeK9-H3 is clearly detected on the uraSE target, the 300-bp region upstream and the ura 4 promoter itself. In the absence of Swi6 diMeK9$\mathrm{H} 3$ persists at uraSE but is lost from the adjacent region and the promoter (Fig. 2B). Thus, as at the mating-type locus (40), Swi6 is required to spread silent chromatin formation outward from the 280-bp uraSE "nucleation center." Because RNAi and/or PTGS clearly remains operative in swi6 $\Delta$ cells, the presence of homologous siRNAs must not be sufficient to mediate spreading. The most parsimonious explanation is that uraSE siRNAs target nascent ura4 transcripts and, in the process, recruit $\mathrm{Clr} 4$ histone methyltransferase, which methylates $\mathrm{K} 9-\mathrm{H} 3$ on local chromatin and thus allows binding of Swi6, which in turn re- cruits Clr4 independently of RNAi to methylate and spread over adjacent nucleosomes (Fig. 6).

The RNAi pathway is involved in the regulation of differentially expressed genes in a number of systems by means of short temporal or micro-RNAs (46-48). Our analyses also demonstrate that RNAi-dependent chromatin silencing acts to repress some genes that lie in close proximity to some retrotransposon LTRs and that are normally induced during sexual differentiation. Together these data are consistent with a scenario where transcripts, homologous to both strands of the LTR, are acted on by the RNAi pathway to generate siRNAs, which cause the nucleation of a patch of diMeK9-H3, Swi6-bound silent chromatin, which can spread outward to silence adjacent genes (Fig. 6). The generation of siRNA homologous to shuraSE depends on Ago1, Dcr1, Rdp1, and $\mathrm{Clr} 4$. Although we have not detected siRNA homologous to Tf1 or Tf2, their generation is clearly implied by this silent chromatin's dependence on these components. More complex mechanisms may account for silencing of RNAiresponding genes located several kilobases from an LTR; for example, long, noncoding transcripts originating in the LTR may interfere with the accumulation of transcripts from these genes and may mediate chromatin-based silencing. Alternatively, the effect may be indirect, owing to activation of some key meiotic transcription factor induced in the absence of RNAi, Clr4, or Swi6. Further detailed investigation will be required to distinguish been these and other possibilities. Nevertheless, the fact that some LTRs elicit a direct effect on neighboring genes is indisputable; RNAi is required for chromatin modification over the LTR adjacent to the D11.02 and meu6 genes and deletion of these LTRs leads to their constitutive expression. Thus, a similar mechanism of RNAi-dependent chromatin silencing appears to mediate the formation of silent chromatin at fission yeast centromeres and the repression of some genes that are induced during sexual differentiation, and this machinery can recognize a synthetic dsRNA and mediate silent chromatin formation on homologous DNA sequences (Fig. 6).

Britten and Davidson proposed that subfamilies of related repeats might regulate the differential expression of distinct networks of genes (14). Here we have demonstrated that full repression of at least two meiotically induced genes requires nearby LTRs, and our data are consistent with other LTRs playing a similar role in regulating other meiotic genes. A recent study has subdivided fission yeast LTRs into nine clades (31); we speculate that these distinct but related LTR families might be coregulated to mediate repression of different gene sets. Coregulation of nonoverlapping gene sets could be achieved with a minimal degree of homology being required to trigger RNAi-dependent chromatin silencing by an siRNA at a particular LTR. Indeed, siRNAs need to be highly homologous to recognize and destroy their RNA target (49); therefore, siRNAs 
identical in sequence to one LTR family would only bring about silent chromatin assembly at other member LTRs and not at nonmember LTRs where the sequences have drifted substantially to form a distinct family. Indeed some Tf1 LTRs are less than $60 \%$ identical, with the longest stretches of identity being less than 20 nucleotides (31).

Analyses in other systems indicate that RNA and protein levels are regulated by endogenous micro-RNAs (46-48). It is possible that some of these also trigger chromatin modifications that allow stable propagation of the silent state through subsequent divisions. Related to this, many studies in metazoans use RNAi for functional analyses of a gene of interest (32). It is possible that not only is the target RNA degraded but that unanticipated chromatin modifications are induced at that genes locus that influence the outcome and interpretation of the result.

Our analyses shows that the mechanism of heterochromatin formation at centromeres and transposable elements are closely related and adds further weight to the idea that ancient transposable elements have been co-opted into centromere structure and function (50). In mammalian cells, there are also similarities between genesilencing mechanisms, retrotransposon silencing, and centromeric satellite-repeat heterochromatin (9, 15-17, 51-53). Whether the RNAi pathway affects these processes in a manner similar to that described here remains to be determined.

References and Notes

1. B. A. Sullivan, M. D. Blower, G. H. Karpen, Nature Rev. Genet. 2, 584 (2001).

2. G. H. Karpen, R. C. Allshire, Trends Genet. 13, 489 (1997).

3. G. P. Copenhaver et al., Science 286, 2468 (1999).

4. C. X. Zhong et al., Plant Cell 14, 2825 (2002).

5. X. Sun, H. D. Le, J. M. Wahlstrom, G. H. Karpen, Genome Res. 13, 182 (2003).
6. P. Bernard et al., Science 294, 2539 (2001).

7. N. Nonaka et al., Nature Cell Biol. 4, 89 (2002).

8. A. M. Weiner, Curr. Opin. Cell Biol. 14, 343 (2002).

9. M. Speek, Mol. Cell. Biol. 21, 1973 (2001).

10. K. Kashkush, M. Feldman, A. A. Levy, Nature Genet. 33, 102 (2003)

11. N. C. Comfort, Trends Genet. 17, 475 (2001).

12. J. A. Jeddeloh, J. Bender, E. J. Richards, Genes Dev. 12, 1714 (1998).

13. A. Miura et al., Nature 411, 212 (2001).

14. R. J. Britten, E. H. Davidson, Science 165, 349 (1969).

15. C. Maison et al., Nature Genet. 30, 329 (2002).

16. M. A. Hakimi et al., Nature 418, 994 (2002).

17. Y. Kondo, J. P. Issa, J. Biol. Chem. 278, 27658 (2003).

18. I. C. Waizenegger, S. Hauf, A. Meinke, J. M. Peters, Cell 103, 399 (2000).

19. R. C. Allshire, E. R. Nimmo, K. Ekwall, J. P. Javerzat, G. Cranston, Genes Dev. 9, 218 (1995).

20. J. F. Partridge, B. Borgstrom, R. C. Allshire, Genes Dev. 14,783 (2000)

21. S. Rea et al., Nature 406, 593 (2000).

22. J. Nakayama, J. C. Rice, B. D. Strahl, C. D. Allis, S. I. Grewal, Science 292, 110 (2001).

23. A. J. Bannister et al., Nature 410, 120 (2001).

24. J. F. Partridge, K. S. Scott, A. J. Bannister, T. Kouzarides, R. C. Allshire, Curr. Biol. 12, 1652 (2002).

25. B. J. Reinhart, D. P. Bartel, Science 297, 1831 (2002)

26. T. A. Volpe et al., Science 297, 1833 (2002).

27. P. Provost et al., Proc. Natl. Acad. Sci. U.S.A. 99 16648 (2002).

28. I. M. Hall, K. Noma, S. I. Grewal, Proc. Natl. Acad. Sci. U.S.A. 100, 193 (2003)

29. T. A. Volpe et al., Chromosome Res. 11, 137 (2003)

30. H. Nakagawa et al., Genes Dev. 16, 1766 (2002).

31. N. J. Bowen, I. K. Jordan, J. A. Epstein, V. Wood, H. L. Levin, Genome Res., in press.

32. G. J. Hannon, Nature 418, 244 (2002).

33. M. Wassenegger, S. Heimes, L. Riedel, H. L. Sanger, Cell 76, 567 (1994).

34. L. Jones et al., Plant Cell 11, 2291 (1999).

35. M. F. Mette, W. Aufsatz, J. van der Winden, M. A. Matzke, A. J. Matzke, EMBO J. 19, 5194 (2000).

36. A. Hamilton, O. Voinnet, L. Chappell, D. Baulcombe, EMBO J. 21, 4671 (2002).

37. D. Zilberman, X. Cao, S. E. Jacobsen, Science 299, 716 (2003).

38. K. Ekwall, G. Cranston, R. C. Allshire, Genetics 153, 1153 (1999).

39. Expression of shuraSE silences $u r a 4^{+}$expression from the wild-type $u r a 4^{+}$locus, from Rint:ura $4^{+}$, and from ura $4^{+}$inserted at bub1, dma1, $\operatorname{mad} 2, \operatorname{rad} 1, \operatorname{rad} 2, \operatorname{rad} 3$, $\operatorname{rad22}$, and wee 1 loci. It is therefore unlikely that flank- ing chromatin makes $u r a 4^{+}$prone to silencing. V. Schramke, R. Allshire, unpublished observations.

40. I. M. Hall et al., Science 297, 2232 (2002).

41. J. Mata, R. Lyne, G. Burns, J. Bahler, Nature Genet. 32, 143 (2002).

42. T. Watanabe et al., Nucleic Acids Res. 29, 2327 (2001).

43. A. V. Gendrel, Z. Lippman, C. Yordan, V. Colot, R. A. Martienssen, Science 297, 1871 (2002).

44. www.genedb.org/genedb/pombe

45. D. C. Weaver, G. V. Shpakovski, E. Caputo, H. L. Levin, J. D. Boeke, Gene 131, 135 (1993).

46. V. Ambros, Cell 107, 823 (2001).

47. D. Baulcombe, Science 297, 2002 (2002).

48. C. A. Kidner, R. A. Martienssen, Trends Genet. 19, 13 (2003).

49. S. M. Elbashir, J. Martinez, A. Patkaniowska, W. Lendeckel, T. Tuschl, EMBO J. 20, 6877 (2001).

50. R. K. Dawe, Plant Cell 15, 297 (2003).

51. F. Sleutels, R. Zwart, D. P. Barlow, Nature 415, 810 (2002).

52. C. Fournier et al., EMBO J. 21, 6560 (2002).

53. C. Tufarelli et al., Nature Genet. (2003)

54. shuraSE is expressed from the nmt41 promoter on the previously described PREP41 plasmid. Transcriptional repressors turn off this promoter in the presence of thiamine. High expression occurs in media with no thiamine.

55. A. F. Dernburg, G. H. Karpen, Cell 111, 159 (2002).

56. We thank members of the Allshire lab for advice and suggestions; A. Pidoux, A. Bird, and N. Hastie for comments on the manuscript; T. Volpe and R. Martienssen for strains and discussions; A. Hamilton and M. Matzke for siRNA detection protocols. We are grateful to $\mathrm{V}$. Wood and $\mathrm{H}$. Levin for communicating data before publication. R.A. also thanks members of the Epigenetic Regulation Network (EC HPRN-CT-2000-00078) for valuable input especially $\mathrm{T}$. Jenuwein for antiserum for methyl H3. This research is supported by the Wellcome Trust. R.A. is a Wellcome Trust Principal Research Fellow.

Supporting Online Material

www.sciencemag.org/cgi/content/full/1086870/DC1

Materials and Methods

Table S1

References

15 May 2003; accepted 7 July 2003

Published online 17 July 2003;

$10.1126 /$ science. 1086870

Include this information when citing this paper.

\title{
Coherent Spin Transfer Between Molecularly Bridged Quantum Dots
}

\author{
Min Ouyang and David D. Awschalom*
}

\begin{abstract}
Femtosecond time-resolved Faraday rotation spectroscopy reveals the instantaneous transfer of spin coherence through conjugated molecular bridges spanning quantum dots of different size over a broad range of temperature. The room-temperature spin-transfer efficiency is $\sim 20 \%$, showing that conjugated molecules can be used not only as interconnections for the hierarchical assembly of functional networks but also as efficient spin channels. The results suggest that this class of structures may be useful as two-spin quantum devices operating at ambient temperatures and may offer promising opportunities for future versatile molecule-based spintronic technologies.
\end{abstract}

Semiconductor quantum dots (QDs) are attractive candidates for scalable solid-state implementations of quantum information processing based on electron spin states (1). Confined car- riers in QDs are relatively decoupled from their dissipative environment as compared with systems of higher dimensionality, such as quantum wells and bulk semiconductors, which are ex- pected to inhibit spin relaxation processes and result in extended spin coherence times. Recently, coherence times of isolated spins in an ensemble of chemically synthesized QDs were found to exceed several nanoseconds, limited by inhomogeneous broadening, up to room temperature (2). In this context, a variety of QD-based quantum computation schemes have been suggested in which efficient and tunable spin coupling among QDs is a crucial implementation requirement (3-8). Although scalability and integration of the proposed devices based on conventional top-down fabrication methods remain challenging, colloidal chemistry techniques permit the creation of semiconductor QDs with controllable size and shape (9). These techniques also allow for chemical reactivity to direct the assembly of the nanometer-scale building blocks into ordered arrays by the judicious choice of the coordinating-ligand functionality. 